\title{
Simulación de procesos sociales basada en agentes software
}

\author{
Millán ArRoyo MEnÉndeZ \\ Dpto. Sociología IV. Universidad Complutense \\ millan@cps.ucm.es \\ SAMER Hassan Collado \\ Dpto. Ingeniería del software e inteligencia artificial. Universidad Complutense \\ samer@fdi.ucm.es
}

Recibido: 23.10 .2007

Aceptado: 19.12 .2007

\section{1. ¿QUÉ ES LA SIMULACIÓN SOCIAL BASADA EN AGENTES SOFTWARE?}

El propósito de este artículo es el de dar a conocer a sociólogos y científicos sociales en general en qué consiste la simulación social, sus posibilidades y limitaciones para la investigación en sociología, partiendo de nuestra experiencia como miembros del Grupo de Agentes Software, Ingeniería y Aplicaciones de la Universidad Complutense de Madrid (GRASIA). Debemos empezar reconociendo que algunas de esas posibilidades y limitaciones las estamos todavía descubriendo, y entendemos que este proceso en marcha, apenas empezado, no culminará hasta haber alcanzado una mayor experiencia en simulación, algo de lo que aún no podemos presumir.

Nuestra experiencia se basa en que el equipo de GRASIA está comenzando a aplicar los conocimientos y experiencias adquiridos en simulación con agentes en otros ámbitos en el desarrollo de una herramienta para la simulación en ciencias sociales. Dicha herramienta consistirá en un software que permitirá a los sociólogos elaborar simulaciones sin necesidad de depender de un experto en inteligencia artificial ni de tener conocimientos ni habilidades de programación. Dicho propósito es innovador, no existe aún ninguna herramienta de este tipo. Para empezar a desarrollarla es imprescindible primero elaborar un modelo de simulación (primero uno, luego más de uno) que parta de lo concreto, de necesidades reales de la sociología actual y sea extrapolable a otros ámbitos y proble- 
máticas que habitualmente investigan los sociólogos. Paralelamente tenemos previsto generar recursos informáticos que se irían aprovechando y reutilizando en cada experiencia de simulación con el objeto de avanzar en la consecución del objetivo descrito. Nuestro primer objetivo consiste en abordar una problemática sociológica en toda su complejidad, y desarrollar los recursos necesarios para poder modelar informáticamente el modelo social previo, pensando que ese desarrollo de recursos, pueda servir para posteriores simulaciones sociales. Más concretamente, estamos pensando en hacer aportaciones que puedan ser útiles en el ámbito de la opinión pública, actitudes y valores de las sociedades reales actuales. Es decir, en el ámbito tradicionalmente vinculado a la investigación demoscópica.

Pero antes de seguir, es necesario explicar qué es una simulación, en qué se basa una simulación con agentes y en qué se diferencia de técnicas de simulación anteriores.

Podríamos definir «simulación» como «el proceso de diseñar un modelo de un sistema real y llevar a término experiencias con él, con la finalidad de comprender el comportamiento del sistema o evaluar nuevas estrategias -dentro de los limites impuestos por un cierto criterio o un conjunto de ellos-para el funcionamiento del sistema» (Shannon, 1975). Nuestra definición alternativa, más comprensiva, la definiría como la emulación del comportamiento de un sistema real por otro artificial, haciendo especial referencia a la posibilidad de realizar experimentos en un sistema artificial (en un entorno controlado, como un laboratorio o un ordenador), el cual se ha modelado previamente para que se comporte de forma análoga al sistema real. La Simulación Social no sería más que un caso particular de ésta, en el que el objeto modelado y simulado es un proceso social.

\section{EVOLUCIÓN DE LAS TÉCNICAS DE SIMULACIÓN HASTA LOS SISTEMAS MULTI-AGENTE}

El estudio de la realidad mediante el proceso de modelado y simulación se ha venido realizando por las ciencias experimentales en los laboratorios desde hace siglos. Podemos situar sus comienzos con las ecuaciones diferenciales en el siglo XVIII, y algo más tarde con los procesos estocásticos (Troitzsch, 1997). Pero los modelos más directamente relacionados con las simulaciones actuales no surgen hasta la primera mitad del siglo Xx con la teoría de juegos, área de la matemática aplicada que utiliza modelos para estudiar interacciones en estructuras formalizadas de incentivos y llevar a cabo procesos de decisión (Davis, 1971).

Otro hito fundamental, surgido de la matemática y la física, son los autómatas celulares, modelos matemáticos que simulan sistemas dinámicos que evolucionan en pasos discretos. Consiste en una rejilla o cuadriculado, donde cada celda de la cuadrícula se conoce como «célula». Cada una posee, en cada 
momento, un estado seleccionado de un número finito de estados posible. Además, cada célula tiene una «vecindad», un conjunto finito de células en las cercanías de la misma. De esta forma, se aplica a todas las células (de forma homogénea, y en cada paso discreto de tiempo) una función de transición que, en base a los valores de la célula en cuestión y los valores de sus vecinos, devuelve el nuevo estado que tendrá dicha cuadrícula en la siguiente etapa de tiempo (Von Neumann, 1966). Las cuatro áreas mencionadas (en cursiva) darán origen a los distintos tipos de simulación «avanzada», computerizada.

Con el desarrollo de los ordenadores en las universidades de los años sesenta comenzó la simulación por ordenador. En un principio se centró en la Dinámica de Sistemas, que requería de la gran capacidad de cálculo que estos ordenadores proporcionaban, ya que consistía en utilizar grandes sistemas de ecuaciones diferenciales para representar las trayectorias de las variables en el tiempo (Hanneman, 1988). Sin embargo, la dinámica de sistemas está restringida al análisis del nivel «macro-social», y solo permitía modelar aquello que pudiera ser traducido a ecuaciones.

A su vez, otros tipos de simulación, derivados de los procesos estocásticos, surgieron o se promovieron con la aparición de los ordenadores, como el modelado de simulación multi-nivel (modelos estadísticos que varían en más de un nivel (Helbing, 1994) o los de teoría de colas (Kreutzer, 1986), respectivamente.

Por otro lado, la conjunción de los autómatas celulares con la teoría de juegos, en el nuevo terreno fértil que proporcionaban las computadoras, permitió desarrollar múltiples usos de la técnica en ciencias sociales. Uno de los más importantes y sin duda el más conocido ejemplo de esta técnica es el juego de la vida, de Conway, (Gardner, 1970) en el que con cuatro reglas muy simples se consigue construir un sistema de autómatas celulares que simula la vida de una sociedad de organismos complejos que interaccionan entre sí. Grandes teóricos de la teoría de los juegos pasaron a implementar problemas sociales con autómatas celulares, como la emergencia de entidades políticas mayores (imperios, alianzas) a partir de la agregación de otras menores (regiones, estados) (Axelrod, 1995) o el voto por mayoría (Capcarrere, 1996).

Pero desde los años noventa surge una nueva técnica del campo de la Inteligencia Artificial y libera a los autómatas celulares de su rejilla, dándoles la posibilidad de ser autónomos y dirigidos por objetivos: los Sistemas Multi-Agente (SMA) (Gilbert, 2006). Se denomina inteligencia artificial a la ciencia que desarrolla procesos que imitan a la inteligencia de los seres vivos. Inteligencia artificial existía desde los comienzos de la informática, pero sólo ha sido recientemente cuando ha podido aprovechar la gran capacidad de cálculo de los ordenadores actuales para desarrollar la computación distribuida a modo de entidades autónomas e independientes que puedan interactuar entre sí: los llamados «agentes».

No hay una definición formal unificada de lo que es un agente software, si bien una mínima definición comúnmente aceptada es la de «un programa autocontenido que puede controlar sus propias acciones, basado en sus percepciones 
del entorno» (Huhns, 1997). Por otro lado, la mayoría de autores considera que los agentes cumplen al menos las siguientes propiedades:

- Son autónomos (pueden operar sin el control directo de humanos u otros agentes).

- Son interactivos (con habilidad social, relacionándose con su entorno y/o otros agentes), conocimiento local (perciben únicamente parte de su entorno «próximo».

- Con percepción subjetiva, racionalidad limitada.

- Son reactivos (perciben su entorno y responden en consecuencia), y proactivos (pueden tomar la iniciativa, con comportamiento basado en objetivos).

- Además, los agentes tienen el potencial de ser programados para tener adaptación, aprendizaje, planificación sofisticada y lenguaje (computacional).

La Simulación Basada en Agentes, a pesar de su reciente desarrollo, está teniendo un rápido crecimiento. Esto es debido principalmente al potencial que ofrece en particular en los dominios caracterizados por un alto grado de localización (como en las redes sociales, donde los nodos y enlaces son esenciales) y distribución (no centralizado y dirigido por una jerarquía, sino distribuido horizontalmente, aunque es posible introducir capas de decisión), frente al modelado basado en ecuaciones (ya comentado), más apropiado para sistemas centralizados dominados más por leyes físicas que por procesos de información.

\section{EXPERIENCIAS DE SIMULACIÓN EN CIENCIAS SOCIALES CON SMA}

La experiencia está demostrando que la simulación basada en ordenador es muy útil especialmente en aquellos contextos de investigación donde los sistemas son demasiado complejos para la experimentación tradicional, o con dificultades prácticas insuperables. Podemos encontrar numerosos ejemplos de simulación basada en SMA en múltiples campos: física, biología, informática, ecología, agricultura, epidemiología, teoría organizacional, marketing, industria... Pero en las ciencias sociales las aportaciones son más escasas.

Uno de los campos de las ciencias sociales donde más se han desarrollado las aplicaciones ha sido en economía. Por ejemplo, la información contenida en los modelos econométricos ha resultado fácilmente reproducible con SSBA. Así podemos observar ejemplos simples de regateo en situaciones de mercado (López-Paredes, 2002) u otros mucho más complejos, como el modelado de un mercado financiero (Jorion, 2006). También se han desarrollado simulaciones para explicar procesos de sistemas auto-organizados, como los de auto-regulación de los mercados (Ygge \& Akkermans, 1995).

Han sido practicadas simulaciones inspiradas en la teoría de la elección racional y bajo el paradigma BDI, entre las que cabe destacar modelos de com- 
portamiento de colas, como clientes en mostradores, en un banco, o en un aeropuerto, o situaciones similares, en las que cada agente es un cliente que busca ser atendido (Gilbert \& Troitzsch, 2006) u otros escenarios relacionados con la elección racional, (Ibidem) etc. El paradigma BDI (Bratman, 197) consiste en una teoría psicológica, basada en la teoría de la elección racional, que ha sido incorporada al diseño de agentes en la informática y popularizada en numerosas aplicaciones. Consiste básicamente en que los actores orientan sus comportamientos racionalmente, en base a deseos e intenciones (objetivos) y creencias (percepciones de los patrones de la realidad).

Dentro de este mismo paradigma merecen también destacarse otras, como simulación de flujos de tráfico, en los que cada agente es un conductor al volante que tiene que tomar decisiones en un contexto en el que interpreta, y reacciona ante otros conductores y las características del tráfico (El Hadouaj, Drogoul And Espié, 2001). O simulación de situaciones de emergencia, como el caso de emergencia en un aeropuerto en el que cada agente es un individuo huyendo del fuego hasta encontrar la salida, (Burmeister, Haddadi \& Mattilys, 1997).

En el campo de la lingüística es destacable un estudio comparativo basado en teoría de redes sociales (Castelló, 2007), en el marco de la evolución dinámica de lenguas en contacto, en un entorno de competencia lingüística. Entre los estudios políticos sobre conflictos fronterizos un buen ejemplo lo constituye (Cederman, 2003), con un análisis a nivel de superestructuras estatales sobre los agentes, mediante simulación de los cambios en las fronteras a nivel geopolítico. Recientemente están surgiendo distintos estudios de las redes de pares P2P, siguiendo el desarrollo de éstas en Internet. Tenemos por ejemplo, (Bin Yu, 2004) y (Xiong, 2004), donde además se desarrolla el tema de «reputación y confianza» (muy común en este área). Existen estudios genéricos sobre cooperación y competencia (Axelrod, 1997), sobre dinámicas sociales, o sobre emergencia de patrones sociales (Gilbert, 1995 y 2002).

Para avanzar hacia una SSBA adaptada a los problemas sociológicos anteriormente definidos es preciso dar un paso más allá de los antecedentes conocidos, al margen de buscar aplicaciones más vinculadas a la tradición sociológica. En las experiencias conocidas no encontramos antecedentes en el intento de simular una sociedad en la que entran en juego dinámicas de interacción social formadas por opiniones, actitudes, valores, ideologías o mentalidades en general. Sin embargo creemos que es razonablemente posible aplicar la SSBA para el estudio de procesos sociales en este contexto de sociedades y perspectivas que tradicionalmente la sociología ha abordado desde las investigaciones demoscópicas. Para estos propósitos el paradigma BDI no resulta aplicable, o cuando menos debería ser seriamente revisado, pues resulta prácticamente imposible definir los deseos e intenciones que mueven a los sujetos en un contexto que puede ser tan amplio como su vida. Es preciso entender que son multipolares y altamente proteicos. Por otro lado, no resulta claro que resulten operativos (en el caso de que pudieran ser definidos) para modelar comportamientos y estados mentales. 


\section{DESARROLLO DEL SOFTWARE PARA SIMULAR}

Actualmente encontramos diversas opciones de software para implementar los SMA. Construir un SMA es una tarea muy compleja si hay que utilizar las técnicas de programación «clásicas» sin ningún tipo de ayuda. Nuestro criterio al elegir un software ha consistido en conciliar la obtención de buenos resultados con la sencillez de manejo (dos criterios difíciles de reconciliar), ya que esta última puede resultar decisiva para que la simulación sea viable como opción de investigación. En los inicios de estos sistemas, había que elegir entre generar un lenguaje de programación orientado a agentes, o desarrollar un «framework» y un IDE. Por «framework» entendemos un conjunto de librerías, pequeñas colecciones de programas y utilidades ya programadas, que proporcionaran ayudas y recursos al programador para facilitar sus tareas. Por IDE entendemos un entorno de desarrollo de programación, es decir, IDE es la herramienta que permite y facilita escribir el código fuente. Pero en los últimos años se ha desarrollado bastante la ingeniería del software orientada a agentes, con lo que han ido apareciendo distintos programas («plataformas» específicas) que, siguiendo una metodología determinada, integran el diseño orientado a agentes, un framework y el IDE.

De las diversas plataformas para el desarrollo de SMA destacamos las siguientes: MAS-CommonKADS (para metodología CommonKADS), MaSE (orientada a objetos), TROPOS (desarrollo simplificado pero no es totalmente de dominio público) e INGENIAS (extensión de MESSAGE/UML y con generación de código automática). Todas funcionan adecuadamente para las aplicaciones comunes de los SMA encontradas en informática. El uso de una u otra dependerá de la experiencia y las necesidades del ingeniero.

En el caso particular de la SSBA, ésta requiere típicamente de gran cantidad de agentes «simples» (ligeros, con poco procesamiento de datos). Los SMA clásicos (de la informática e industria), en cambio, suelen utilizar relativamente poca cantidad de agentes «complejos» (pesados computacionalmente). Ninguna plataforma de las citadas se adapta perfectamente a las necesidades de la SSBA, por estar enfocadas a los SMA clásicos. Y aunque es posible realizar agentes ligeros con ellas, resulta más complicado que permitan lidiar con grandes números de ellos.

Por otro lado, hoy en día, es el programador (normalmente un ingeniero de software especializado) y no el investigador social el que debe realizar el trabajo de implementación del modelo informático y la simulación. Por tanto, a menos que éste tenga un adecuado conocimiento del modelo sociológico, resulta difícil que el científico social (que normalmente tampoco sabrá nada de programación) le comunique cómo quiere que sea el modelo exactamente. En GRASIA estamos trabajando actualmente así, por necesidad de comenzar a impulsar estas primeras experiencias de simulación social, aunque reconocemos que no es la situación ideal. El caso ideal sería que el sociólogo, conocedor del modelo sociológico, pudiera servirse de esta herramienta de forma autónoma. 
Para ello sólo hay dos caminos. O el sociólogo aprende a programar o se genera un software que le permita modelar y simular de modo análogo a cómo realiza un análisis multivariante mediante un paquete de análisis estadístico, sin necesidad de programar o de conocer en profundidad los algoritmos matemáticos implicados. Hoy por hoy resulta muy conveniente que el sociólogo programe.

Siendo conscientes que la segunda opción es la más adecuada para integrar la simulación en el oficio del sociólogo, nos proponemos como reto desarrollar una plataforma de SMA (partiendo de una pre-existente) que:

a) Soporte adecuadamente los sistemas de SSBA

b) Permita a cualquier científico social utilizarla como usuario para formalizar su modelo sociológico y que sea la plataforma la encargada de generar automáticamente todo el código necesario (el programa correspondiente a ese modelo) y simularlo.

Para ello, nos basamos en la plataforma INGENIAS y en su INGENIAS Development Kit (IDK), que por poseer unas herramientas de meta-modelado, un lenguaje gráfico de modelado de agentes, y un módulo de generación de código muy potentes ha sido la que hemos elegido (Gómez-Sanz). Actualmente la plataforma ya ha incorporado posibilidades de generación de grandes números de agentes, además de personalización para un dominio de aplicación concreto (fundamental en ciencias sociales, donde no tiene sentido buscar el modelo general para todos los dominios). Pero aún no tiene las plantillas necesarias (templates, abstracciones genéricas usadas en informática para saber qué «palabras» colocar dónde) para poder generar código adaptado a la simulación social. Es en este punto donde tiene sentido construir «artesanalmente» (sin generación de código, ya que no está disponible todavía) un sistema de SSBA que no sea demasiado dependiente del contexto, centrándose en un único ejemplo, como otros sistemas vistos anteriormente. En este sistema de simulación social debemos tomar las decisiones adecuadas, tanto de modelado (debe funcionar adecuadamente) como de implementación (debe ser modular y estructurado para poder generalizarlo), y así poder abstraer las plantillas necesarias.

Para implementar «artesanalmente» un SMA de simulación social no existen todavía plataformas completas, sin embargo sí podemos acudir a los mismos recursos que se disponían antes de ellas, ya comentados. Así pues, existen múltiples lenguajes, frameworks e IDEs disponibles para la tarea. Por la forma en que son desarrollados, la elección del lenguaje y del IDE quedan dependientes del framework elegido, mucho más vital a la hora de ayudarnos en nuestra tarea.

En (Railsback, 2006) se comparan en detalle los cinco principales frameworks. NetLogo se evalúa como muy simple; los más rápidos son Repast y Mason (éste último algo mejor, ya que está centrado en este aspecto); los más maduros Repast y Ob-Swarm (este último descartado por arcaico, y Mason destaca por su inmadurez); J-Swarm no destaca en nada. En (Axtell, 2006) se repasan numerosas alternativas, analizando únicamente su dificultad y rendi- 
miento. En este caso los que mejor «puntuados» quedan son Repast (o su versión en desarrollo Simphony), Mason y Ascape 3.0 (comercial, no libre). Siguiendo estos criterios expuestos, para desarrollar nuestro sistema hemos utilizado; Repast como framework, Java como lenguaje (por disponer de muchos más recursos que en otros lenguajes) y Eclipse como IDE.

\section{EL MODELADO DE LA EVOLUCIÓN DE LA RELIGIOSIDAD EN ESPAÑA. MODELO 500}

Hemos elegido simular la evolución de la religiosidad (e irreligiosidad) de la sociedad española desde 1980 hasta el año 2000. La ventaja de este ejemplo es que se conoce suficientemente (a grandes rasgos) y hay información cuantificada (también cualitativa) de dicha evolución, por lo que era factible el ejercicio de introducir información de la situación en 1980 y observar si los resultados de la simulación se ajustaban a la evolución real conocida, con el objeto de validar la bondad de la herramienta en su aplicación a un proceso social más abierto y complejo que los antecedentes de simulación conocidos (y más «sociológico»).

En esta situación, como en muchas otras de la investigación sociológica actual, las teorías de la elección racional tienen poco que aportar, y en concomitancia con esto, el comportamiento de agentes basado en «deseos-creencias-e intenciones» (BDI, el más elegido hasta ahora en las simulaciones sociales) tiene una capacidad limitada (insuficiente o incluso potencialmente distorsionadora, en lo que respecta a las intenciones) a la hora de predecir los comportamientos de los individuos en contextos más amplios que estructuras organizativas relativamente cerradas (empresas, organizaciones burocráticas, mercados de consumo o laborales, comportamiento en colas, o en situaciones de tráfico de automóviles o comportamiento de viandantes, etc.).

Nos resultó necesario en el modelado de agentes y de su entorno imaginar mecanismos determinantes del comportamiento de agentes independientes de sus «intenciones vitales» para poder simular representaciones mentales complejas como (en el caso que nos ha ocupado) los patrones de religiosidad, u otras (aunque aún no las hemos llevado a cabo, estamos reflexionando sobre cómo hacerlo) como valores, ideología política, o moralidad. Por dos motivos. Primero porque resulta inabordable formular cuales son la metas «vitales» de los individuos (si es que existen), y en segundo lugar porque las metas o intenciones vitales de los agentes en relación con valores, o sistemas narrativos como los discursos políticos o religiosos, son sumamente proteicos. La constante búsqueda de sentido (sentido de la vida) no se ajusta a una intención inicial e inmutable que el sujeto busca, sino que se reelabora constantemente. Nuestros objetivos vitales son en buena parte un producto de nuestra condición social y de la pura contingencia, aparecen mediados por la percepción de nosotros mismos y por las expectativas de los demás. Por otro lado, los seres humanos somos solo en parte seres racionales, y buena parte de nuestras conductas y pensamientos se rigen 
por esquemas emotivos o no racionales. El desarrollo futuro de los SMA aplicado a la investigación sociológica y antropológica, deberá tener mucho más en cuenta estas cuestiones, desde el planteamiento inicial de las cualidades básicas de los agentes. Digamos que hoy existe la tecnología necesaria para poder emular estos procesos de forma razonablemente exitosa, aunque aún debe ser convenientemente desarrollada. A pesar de este inconveniente, hoy por hoy las posibilidades de simulación mediante SMA son importantes, ya que las limitaciones señaladas pueden ser sorteadas en el modelado, por ejemplo introduciendo pautas probabilísticas o semi-probabilísticas de comportamiento en función de datos disponibles. Existen también otros recursos, como la aplicación de lógicas borrosas (Zadeh, 1996) o redes bayesianas.

En nuestro tema elegido, partimos del discernimiento de cuatro grandes patrones (o formas) de religiosidad católica, que hemos denominado: «eclesial», «laxa», «alternativa» y «no religiosa» (Arroyo Menéndez, 2005). Los «eclesiales» son individuos relativamente próximos a la jerarquía (Obispos y Vaticano), son católicos practicantes que confían en la Iglesia y asisten a misa semanalmente. Los «laxos» practican una religiosidad de baja intensidad, con un nivel de práctica sensiblemente más bajo (suelen ser católicos no practicantes que profesan una religiosidad «a la carta», de «aujste existencial»). Son creyentes que confían en la Iglesia y asisten a servicios religiosos ocasionalmente o nunca (o bien también se incluye un grupo muy minoritario de practicantes semanales que no confían en la Iglesia). Los «alternativos» son personas con fuerte identidad religiosa pero que no confían en la Iglesia y no asisten regularmente a servicios religiosos (en su inmensa mayoría siguen manteniendo su identidad católica). Por último, los «arreligiosos» son ciudadanos que ni confían en la Iglesia ni se consideran personas religiosas, independientemente de que crean o no en Dios (la mayoría son agnósticos o ateos, pero no todos). Sabemos que en los últimos 20 años los eclesiales han disminuido considerablemente, mientras que han aumentado los arreligiosos y los alternativos. Los laxos han oscilado aumentando en 1990 y disminuyendo luego en el 2000 alcanzando valores similares a los de 1980.

Hasta ahora hemos desarrollado un primer prototipo de simulación, muy simple, que ya se ha implementado en los momentos en los que se escriben estas líneas (el Modelo 500) mientras estamos en pleno desarrollo de otro más avanzado (Modelo 2300). Pese a la relativa simplicidad de nuestro primer modelo, ya se ha conseguido el objetivo básico de simular de forma realista esta evolución conocida, si bien, para que la herramienta resulte adecuada para la experimentación requiere un modelado mucho más realista y detallado, que es lo que se está consiguiendo en diversas etapas.

Las prioridades del «Modelo 500» son las de programar sólo algunos de los aspectos más básicos de un sistema social. Estos son la estructura demográfica de la población española (tomada desde el momento inicial de 1981) y su evolución dinámica desde entonces. Lo que implica pautar que los agentes tienen amistades, se casan, tienen hijos, socializan a sus hijos en unos valores y luego mueren, todo ello de acuerdo con unas pautas realistas. Una vez implementados 
estos recursos no solo están disponibles para posteriores simulaciones de la misma sociedad, sino de otras, con unos mínimos cambios de los parámetros demográficos. El modelo sociológico subyacente es muy simple; consiste en modelar tan solo uno de los vectores de socialización. Se basa en la teoría de Inglehart (Inglehart, 1998) de que los valores (incluidos los religiosos) se adquieren en una etapa de la vida relativamente temprana, estimada en torno a la adolescencia y primera juventud, siendo en este momento cuando principalmente se forjan los valores, los cuales se mantendrán relativamente estables durante el resto de la vida. Después los cambios son posibles, pero más atenuados. En este contexto es predecible una importante influencia de los valores de los padres en los hijos, de modo que padres no religiosos tenderán a educar hijos igualmente o menos religiosos que los padres, y padres religiosos tenderán a educar hijos más religiosos. Con este primer «Modelo 500» se pretendía explorar la capacidad predictiva (y explicativa en un segundo plano) de la conjunción entre la inercia demográfica (van muriendo los viejos, que son más religiosos y van quedando los jóvenes que cada vez son menos religiosos) y los procesos de socialización en marcha (los hijos reciben la «herencia social» de los valores de sus padres). Es evidente que inciden otros factores que progresivamente se irían acoplando al modelo una vez visto la capacidad explicativa del primero, que hipotéticamente suponemos más importante.

Para modelar esta evolución hemos partido de las características de los españoles y de la sociedad española de 1980. Como es imposible simular una sociedad en su globalidad, debemos conformarnos con una simplificación centrada lo más relevante del proceso social de nuestro interés. Esta simplificación nos pone ante el riesgo de caer en el reduccionismo, pero es inevitable, como también ocurre con otras metodologías. Hemos optado en nuestro primer modelado por crear una sociedad análoga a la española pero conformada por tan solo 500 agentes (de ahí el nombre del modelo) debido a que cuantos menos agentes interactúen más rápidamente se procesarán las simulaciones. En las primeras pruebas es recomendable trabajar con pocos agentes e ir aumentado su número conforme comprobemos que el modelado se está encauzando bien. Dado que nuestro interés era que la sociedad de 500 agentes fuese en sus aspectos cualitativos y cuantitativos análoga a la sociedad de 1980, hemos elegido por muestreo aleatorio simple una submuestra de la Encuesta Europea de Valores (EVS, 1980) llevada a cabo en España, y hemos asignado a los 500 agentes las características de 500 individuos reales, (entrevistados) en las variables elegidas. De modo que de forma casi automática nos aseguramos al asignar características a los agentes la similitud distributiva de dichas características respecto a la sociedad real (propiedad que ya cumplía la encuesta).

Deseamos llamar la atención sobre este procedimiento, por dos motivos. En primer lugar, utilizar una encuesta como patrón de asignación de características de sociedades de agentes simplifica al máximo las tareas de definición y distribución de variables, a la vez que dota al modelo artificial de una base muy realista (si la encuesta es buena, y si en esta se encuentran las variables que buscamos). En se- 
gundo lugar, destacaríamos la interesante posibilidad de complementar el método de encuesta mediante simulación con SMA, con el objeto de estudiar procesos interactivos, algo imposible de hacer hoy mediante métodos cuantitativos clásicos y solo posible mediante técnicas cualitativas grupales. ¿Imaginan poder estudiar las reacciones interactivas de los sujetos entrevistados aisladamente en una encuesta, atendiendo a las múltiples características de las variables contempladas? Lo que explicamos a continuación (nuestra simulación) parte de esto, pero supone tan solo una de las múltiples aplicaciones que cabe imaginar sobre esta posibilidad que se abre. De igual modo, los resultados de análisis de redes sociales realizados mediante software especializado (Pajek y otros) también pueden utilizarse como patrón de la estructura de interacciones de una sociedad dada, siguiendo una red real. Otra ventaja de este procedimiento es que nos ha simplificado al máximo la descripción de las características demográficas de la sociedad española, dado que estaban implícitas en la muestra, con excepción de la programación de la reproducción (nacimientos de hijos) envejecimiento y muerte de los agentes, que han sido diseñadas siguiendo el patrón demográfico de la España real. Por último, debe destacarse la ventaja de que ayuda a reducir al mínimo el número de parámetros, simplificando la programación.

Se han seleccionado previamente sólo unas pocas (las más esenciales) de las variables intervinientes en el proceso. En primer lugar, cabe citar la que podemos tomar como variable «dependiente», esto es, los diversos patrones de religiosidad explicados. En segundo lugar, se han incluido los factores o variables más relevantes para explicar y/o predecir el tipo de religiosidad de los sujetos, en función de modelos de regresión previos, y del conocimiento de las interacciones entre variables partiendo del análisis empírico de los datos de encuesta. (Arroyo Menéndez, 2004, a). Estas variables han sido las características básicas de los agentes. Sexo, edad, estudios, clase social e ideología política, así como algunos indicadores de permisividad moral.

\section{LOS RESULTADOS OBTENIDOS EN LA SIMULACIÓN DEL MODELO 500}

Con tan solo este modelado elemental y burdo se ha conseguido ya una simulación de la evolución de los patrones de religiosidad bastante realista, como reflejan los gráficos y datos que se presentan a continuación, en los que se comparan datos de encuestas de mediciones de 1980, 1990 y 1999 y los resultados de la simulación cuyo input solo tiene en cuenta la situación de España de 1980. De las diversas pruebas de simulación efectuadas (6 en total) se han elegido las dos soluciones más extremas, la «óptima» (la más ajustada a los datos reales) y la pésima (la menos ajustada a los datos reales) ${ }^{1}$.

${ }^{1}$ Las diferencias en 1980 son debidas a que en la simulación damos los datos de la submuetra $(n=500)$ y en «datos EVS» son el total de la muestra. (Obedecen al muestreo aleatorio).

EMPIRIA. Revista de Metodología de Ciencias Sociales. N. ${ }^{\circ}$ 14, julio-dicembre, 2007, pp. 139-161. ISSN: $1139-5737$ 

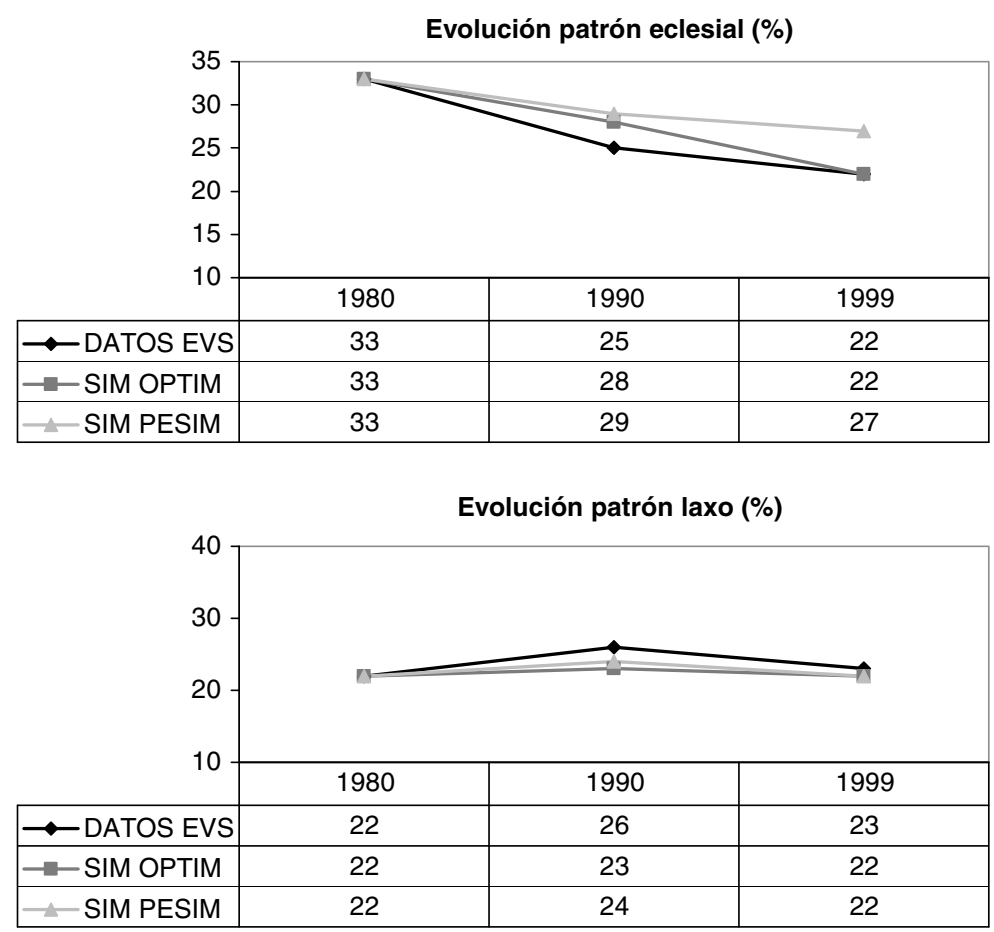

Cualquiera de las simulaciones se ajusta muy bien a los datos conocidos, incluso la llamada «pésima». Las diferentes pruebas responden a distintas modulaciones de las interacciones de los agentes, manipulándose el tamaño del espacio y con este la intensidad de las interacciones entre agentes. El mejor ajuste corresponde a una intensidad de interacciones elevada, mientras que el peor ajuste corresponde con una intensidad más baja.

El motivo de que la simulación funcione con un modelado aún tan precario (no nos esperábamos resultados tan aceptables en este primer prototipo, el cual respondía más a requisitos de la elaboración informática que al modelado sociológico), responde a la conjunción de dos hechos. De un lado a la importancia e intensidad de los cambios intergeneracionales en la evolución de patrones de religiosidad. De otro, a la fuerte inercia del patrón demográfico como factor que permite explicar y predecir aquellos cambios sociales (como los cambios de valores o los cambios en la religiosidad vividos en España) sujetos a fuertes diferencias intergeneracionales, y cuya variación intrageneracional no se acusa tanto. La simulación sirve para constatar la importante capacidad predictiva de las diferencias intergeneracionales y la concomitante dinámica demográfica, en conjunción con las pautas de socialización recreadas. Se trata de algo que ya se había observado en un estudio anterior (Arroyo Menéndez, M. 2004 b) y que 

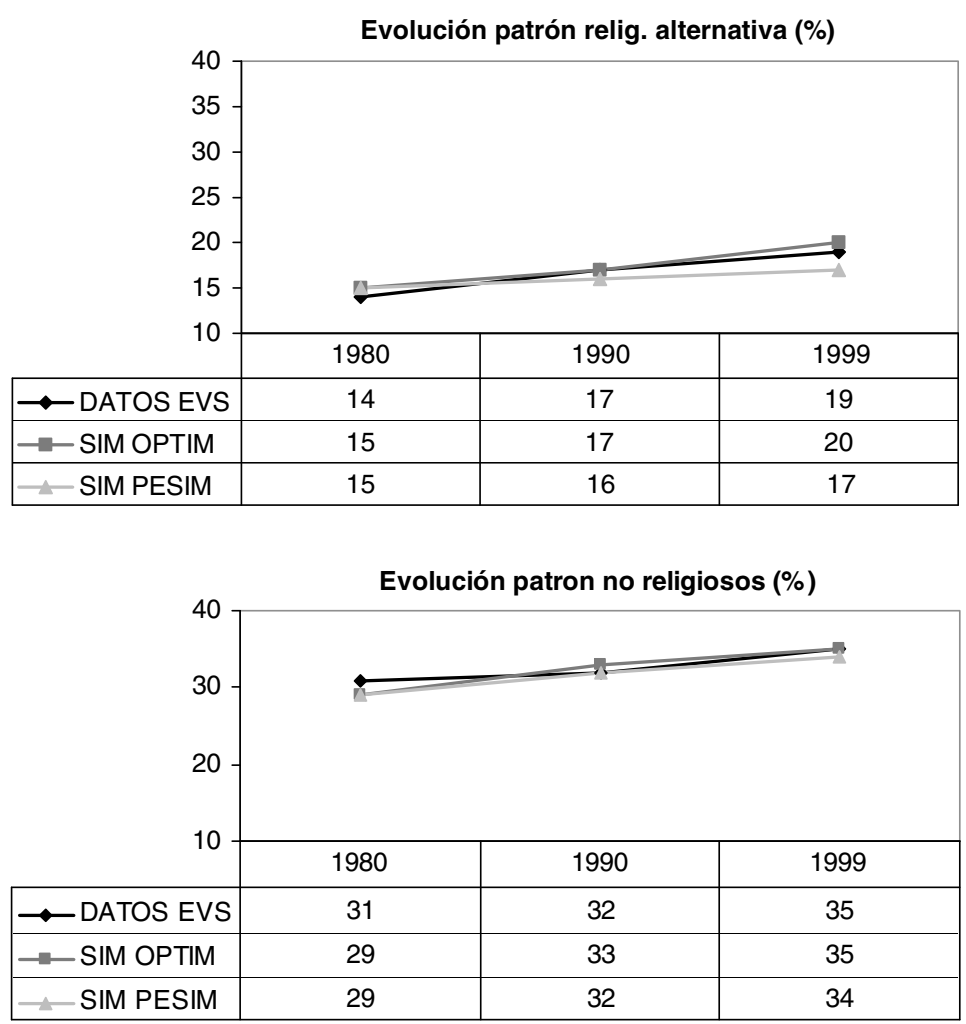

ahora ha resultado incluso mayor de lo que se esperaba. En base a lo cual es razonable predecir para un futuro próximo un alejamiento de la Iglesia mayor que el actual, merced al cual seguirán incrementando los patrones de no religiosidad y de religiosidad alternativa en detrimento de los otros dos. Tan solo teniendo en cuenta la inercia de la conjunción de factores aquí considerados.

Por otro lado nos parece relevante exponer que no solo ha ajustado la religiosidad a los datos recogidos por la encuesta diez y hasta veinte años después, sino en general, han tendido a ajustar razonablemente bien todas las variables consideradas en la simulación, a saber: posición socioeconómica, nivel de estudios, posicionamiento ideológico en la escala izquierda-derecha, y algunos indicadores de permisividad moral: aborto, divorcio, eutanasia, homosexualidad y sexualidad. De la ideología política no se ha llegado a predecir el repunte hacia la derecha que se produce (de acuerdo con las series históricas del Centro de Investigaciones Sociológicas ${ }^{2}$ ) desde mediados de los noventa hasta el 2002, si bien consideramos que era pedir demasiado a unos datos tomados en 1981. Del

\footnotetext{
${ }^{2}$ Pueden consultarse en su web www.cis.es.
} 
resto de las variables (incluida esta última, si exceptuamos dicho repunte) cabe exponer que las tendencias de la simulación coinciden en líneas generales con las tendencias que se han observado en los datos empíricos recogidos por encuestas.

Todo esto nos está indicando que la capacidad predictiva de la edad, conjugado con la dinámica demográfica y el modelo de socialización, es muy alta al menos en el periodo de tiempo contemplado (10-20 años), no solo en lo referido a la evolución de la religiosidad, sino en un espectro más amplio de fenómenos, entre los que por supuesto se incluyen los valores, la moral y la ideología política.

Aparentemente los datos de una única medición se comportan razonablemente bien a veinte años, sin necesidad de acumular ni proyectar ninguna serie temporal, y sin poner todavía en marcha todo el potencial de los agentes. Lo desarrollado hasta ahora (aunque se puede y se debe mejorar) sienta las bases para una buena proyección en el tiempo de datos transversales (como los de una única encuesta), aplicados al menos a valores o rasgos de mentalidad relativamente estables, más allá de los considerados en esta aplicación concreta.

\section{EL MODELO 2300}

Quedaban aún muchas otras variables por incorporar en el modelo implementado de las que se conoce previamente su valor predictivo, explicativo o teórico. En el modelo 2300 (hemos ampliado el número de agentes al total de la muestra española de la EVS 1981, $n=2300$ ) estamos ya incluyendo las que consideramos las principales variables que interaccionan con la religiosidad. Este es el caso de los valores, dado que los relacionados con la individualización social (HALMAN, 1995) son especialmente importantes como predictores de la religiosidad y de la explicación teórica del descenso de influencia de la Iglesia sobre el individuo (ARROYO MENÉNDEZ, 1994 a). Desde el modelo sociológico que se articula (basado en los desarrollos de los equipos de las encuestas Europea y Mundial de Valores) los valores asociados a la modernización cultural: individualización social (Halman, 1995) y postmaterialismo (Inglehart, 1977, 1991 y 1998) tienden a distanciar a los individuos de la religiosidad e influencia de la Iglesia, y viceversa, los valores religiosos fomentados desde la institución eclesial constituyen un freno al desarrollo de la modernización cultural secularizada. Además se están incluyendo un conjunto amplio de variables de religiosidad y no sólo una variable (creencias, prácticas, actitudes ante la Iglesia) e indicadores de permisividad moral (aborto, divorcio, eutanasia, sexualidad...).

También están siendo incluidos otros objetivos básicos, como modelar la estructura de interacciones entre los agentes y las pautas que les permiten a cada uno evolucionar en el tiempo (en el modelo anterior las características eran todavía estáticas y por tanto no se aprovechaban las posibilidades tecnológicas de los SMA. Al finalizar la actual etapa sí se conseguirá).

El modelo sociológico implícito en las pautas de interacción considera que los agentes interactúan preferentemente con individuos con características se- 
mejantes, aunque no iguales; semejante clase social, edades semejantes sobre todo siendo jóvenes, preferencia por la proximidad en el espacio físico y tendencia a no ser muy diferentes en sus valores. Las redes de interacción se tejen en torno a estos preceptos sumamente elementales (en posteriores desarrollo esperaríamos que los análisis de redes sociales permitan desarrollar modelados más finos, realistas y complejos). Luego se definen diferentes clases de interacciones; a un nivel más superfluo están las interacciones con conocidos (menos intensas), luego con amigos, más intensas y menos numerosas, luego especialmente intensas con la pareja (con la cual luego se procrea y se forma la unidad familiar). Además están las relaciones entre padres e hijos, especialmente importantes en el proceso de socialización pero no del todo decisivas en este modelo.

Una importante fuente de cambios proviene de las interacciones entre agentes. Los agentes que más interactúan más probabilidad desarrollan de influirse recíprocamente, acercando mutuamente posturas. Siguiendo la conocida teoría de Inglehart estamos considerando que los individuos más jóvenes (por debajo de los 20 años) sí pueden estar probabilísticamente expuestos a bruscos cambios de valores y de mentalidad en general (y en su religiosidad en particular) en función del entorno ambiental, pero después no se admiten cambios bruscos sino moderados o pequeños, en función de los valores mantenidos por las personas con las que más se interacciona (asumiendo el supuesto de que los individuos en fuerte interacción tienden a aproximar sus valores y actitudes).

$\mathrm{Al}$ igual que ocurre con los valores en general, no se permitirá a los agentes cambiar bruscamente de patrón religioso (las conversiones o desconversiones súbitas son muy infrecuentes en la vida real). Siguiendo un modelo de evolución empíricamente observado, se admiten ciertas variaciones ligeras por efecto del ciclo de vida, de manera que en el paso de la adolescencia a la juventud es frecuente que la religiosidad disminuya moderadamente. De ahí en adelante el nivel de religiosidad del sujeto muestra una tendencia a permanecer relativamente estable, o a cambiar de forma moderada y progresiva, en su alejamiento, pero también en su acercamiento al paradigma eclesial, dependiendo del entorno social y de los ajustes de mentalidad de los sujetos.

Por otro lado, otra fuente importante de cambios que ahora contemplamos proviene de la propia reflexión lógica del sujeto, de su discurso, de sus racionalizaciones. En esta simulación del pensamiento estamos combinando el uso de probabilidades con reglas de coherencia lógica. A veces usamos unas y otras veces las otras. Por ejemplo, se están estableciendo pautas para evitar ciertas situaciones de «disonancia cognoscitiva» que pueden generase en proceso interactivos. Por ejemplo, dado que es improbable (aunque no imposible) que un individuo asuma una posición muy de derechas y sea a la vez anticlerical, se le fuerza probabilísticamente a decantarse por una de las dos opciones y en consecuencia reformular o su ideología política (que deberá suavizarse mucho convergiendo hacia el centro) o su patrón religioso, que oscilará bien hacia el perfil «laxo» o hacia una religiosidad «alternativa» según el caso. De la misma manera, está previsto implementar el sistema para que si una persona se divorcia o tie- 
ne un embarazo no deseado, o vive sin casarse, etc., tenga que reformular su religiosidad, alejándose de la religiosidad eclesial, para conciliar actitudes y comportamientos, tanto ellos como las personas de su más inmediato entorno. (Se introducirían en principio aleatoriamente este tipo de sucesos, con una probabilidad proporcional a su ocurrencia real).

El lector debe advertir que estas soluciones, una vez desarrolladas y comprobada su capacidad predictiva y explicativa, no sólo son útiles en el tema actual, sino son utilizables en situaciones muy diversas, con unos cambios mínimos (de parámetros nada más). Los mayores esfuerzos de modelado y los tiempos más dilatados de ejecución se están realizando precisamente ahora. En la medida que se avance, se irá más rápido y los modelados de la sociedad española (o de otros países) será más rápida, más flexible y más realista. Una vez se hayan acumulado abundantes recursos de programación, estaríamos en condiciones de generar un software para que el modelador sea un simple usuario que no necesite programar.

\section{LIMITACIONES ACTUALES Y DESAFÍOS FUTUROS}

En la fase de trabajo en la que nos encontramos, se hace evidente la principal dificultad de la aplicación de la simulación social a la sociología, que no recae sobre la vertiente informática sino sobre la sociológica, esto es, el gran desconocimiento existente de fenómenos sociales básicos, tanto por carencia de evidencias empíricas de un lado, como de otro por limitaciones de la teoría sociológica. Por ejemplo, al definir las pautas de interacción, nos encontramos con la carencia de estudios sistemáticos que arrojen luz sobre aspectos concretos sobre cuántos amigos frecuentemente se tiene, sobre la probabilidad de un sujeto de emparejarse con otro de distinta clase social, o sobre las influencias recíprocas en nuestros valores, actitudes y opiniones de las personas con las que habitualmente interaccionamos en nuestra vida cotidiana. Apenas se sabe cómo se produce o se ha producido y en qué medida la transmisión de los valores y creencias objeto de nuestro estudio (en especial los religiosos y/o los relacionados con estos), en la familia y en otros grupos primarios, o el efecto de los medios de comunicación social. Se conoce aún muy poco (pese al creciente desarrollo actual) sobre la emergencia y estructuración de redes sociales, que permitan definir los patrones de interacciones «macro» $\mathrm{y}$ «micro» que intervienen en un proceso social concreto, así como su posible impacto en la transmisión y cambio de valores, actitudes u opiniones. Tampoco conocemos bien ni tenemos apenas datos que ayuden a modelar a nivel micro los procesos de cambio en las creencias y valores de los individuos. Estos son conocimientos básicos que no solo son aplicables a este modelo concreto sino a muchos otros de interés sociológico, como serían, procesos de cristalización y cambio de la opinión pública, actitudes y comportamientos sociopolíticos, comportamiento de consumidores, etc.

No obstante lo anteriormente expuesto, la simulación social puede ser un buen pretexto para tomar conciencia de estas carencias y tratar de superarlas. Es 
decir, al margen de lo que nos permita descubrir por sí misma, al superar las barreras de la experimentación en las ciencias sociales, y mucho antes de haber llegado a tal objetivo, nos pone ante la necesidad de formular modelos explicativos mucho más formales, elaborados, documentados y sistemáticos de los que los sociólogos acostumbran.

\section{APORTACIONES POTENCIALES DE LA SSBA A LA SOCIOLOGÍA}

Aunque el modelado informático es un mero instrumento al servicio del sociólogo, en nuestra opinión y experiencia ayuda mucho a pensar y formalizar el modelado sociológico. Primero por la necesidad de alcanzar una visión global (sistémica) de un proceso social en su contexto dinámico e interactivo con otros hechos y procesos sociales. Segundo, porque al requerir una mayor precisión que el lenguaje natural que en muchas ocasiones (no siempre) utiliza el sociólogo, fuerza al desarrollo de modelos explicativos más precisos y formales que los habituales, y lo que quizás sea más interesante, nos ayuda a combinar diversas explicaciones, teorías y datos, a formularnos preguntas que quizás anteriormente no nos habíamos planteado.

La propia combinación de teorías y datos, permite alcanzar una visión sociológica desde una mayor perspectiva. Por ejemplo, en el caso que nos ocupa, estamos teniendo en cuenta (y/o pensamos tener en cuenta en próximos desarrollos) datos empíricos de las colecciones de encuestas de valores, informaciones provenientes de otras encuestas de religiosidad, informaciones provenientes de estudios cualitiativos, marcos teóricos provenientes del campo de la sociología de la religión (religiosidad invisible, privatización de la religiosidad, transmisión y «olvido» de la memoria religiosa, y cambio religioso como consecuencia del avance del proceso de modernización), teorías sobre el cambio de valores en las sociedades industriales avanzadas de los equipos de las encuestas mundial y europea de valores, etc.

La combinación de datos y diversos marcos teóricos no solo se puede hacer mediante la simulación social, pues no es infrecuente que los sociólogos combinen diversas perspectivas teóricas para abordar empíricamente temáticas concretas. Pero esta tiene la ventaja adicional de que ofrece un marco para comprobar si la articulación (sociológica) que hemos desarrollado «funciona», mediante las diversas pruebas de simulación que puedan establecerse en el modelo, cuando se ponen en interacción los agentes, y observamos si las reglas que hemos introducido permiten resultados análogos a los conocidos en la sociedad real.

Por otro lado, cuando no existen datos o teorías comprobadas, existe la posibilidad de modelar algún o algunos aspectos concretos en base a nuestras propias hipótesis. Esta posibilidad constituye una de las principales razones por las que un sociólogo se puede interesar por la simulación social; someter a prueba una hipótesis sin necesidad de recrear situaciones de experimentación empíricas (a 
menudo imposible en ciencias sociales). Cuando un modelo está suficientemente desarrollado, se puede probar a introducir un aspecto del que carecemos de información con datos o normas hipotéticas, y probar para ver si la simulación mejora o no la emulación del proceso social real, según datos conocidos.

Precisamente esta es la estrategia que estamos siguiendo (y/o que pretendemos seguir) para suplir las carencias anteriormente señaladas. Se modela en base a supuestos hipotéticos, observamos si la simulación mejora o empeora los resultados, y jugamos con los parámetros o reglas para determinar el ajuste más óptimo a los datos en todo el sistema. Cuando estamos seguros de que el modelo «funciona» (se comporta de acuerdo a los datos empíricos conocidos o a teorías refutadas) vamos introduciendo nuevas hipótesis para someterlas a prueba, observando si el modelo mejora o empeora de acuerdo con lo ya conocido y comprobado.

Una interesante variante de esta posibilidad de suplir carencias de información tiene lugar cuando contamos con una información cualitativa previa de la que desconocemos su cuantificación. Podemos, por ensayo y error, ir modificando parámetros hasta observar cual de estos produce mejores ajustes de acuerdo con los datos reales conocidos. Es decir, permite algo tan interesante como cuantificar lo cualitativo (al menos en términos de aproximación) sin necesidad de ejecutar trabajos de campo reales. Insistimos en que esto sólo arrojaría resultados realistas en el supuesto de un modelo muy desarrollado, pero la posibilidad de hacerlo está ahí.

Utilizando la inteligencia artificial de los SMA no es necesario (aunque también lo admite si se considerase oportuno) modelar a base de ecuaciones o sistemas de ecuaciones, como es el caso de otros métodos de simulación y otras formas de elaborar modelos cuantitativos (modelos econométricos, de análisis multivariente, de ecuaciones estructurales, etc.). Esto es debido a que la inteligencia artificial de cada agente deviene en un comportamiento emergente global, actuando como base para definir la estructuras de interacciones de las variables o factores implícitas en dichas características, de modo que la conjunción de esta interactividad y sus características (más las reglas que definamos de actuación en función de características) sustituyen la función de las ecuaciones en otros modelos cuantitativos. Las representaciones mediante modelos de simulación acostumbran a ser más similares a los procesos del mundo real que los provenientes de modelos matemáticos. (Lozares, 2004).

Esta propiedad adquiere especial interés en situaciones en las que en nuestros modelos teóricos postulamos (o sospechamos), más que relaciones de causalidad unidireccionales, relaciones de intercausalidad, es decir, cuando las variables dependiente e independiente alternan sus papeles intermitentemente. Esto es muy frecuente en ciencias sociales. Concretamente en el caso que nos ocupa, podemos postular que los cambios religiosos están en función del cambio en los valores, pero también puede ser razonable postular lo contrario.

Trabajar bajo el paradigma de agentes resuelve los problemas de establecer las direcciones de causalidad, precisamente porque no necesitamos hallar ecuaciones, y por tanto no necesitamos definir previamente cual es la variable inde- 
pendiente y cual es la dependiente (a menudo la consideración es falsa o meramente aproximativa). Entendemos que existe un importante grado de retroalimentación entre religiosidad y posiciones ideológicas y es mayor aún entre religiosidad y determinadas dimensiones de los valores, a la vez que estos interaccionan también con la ideología política. Lo que se produce es más bien un proceso de reajuste potencialmente multidireccional del patrón global de la mentalidad del sujeto, de manera que cuando se producen cambios en una dimensión se reajustan también otras. Por ejemplo, si un individuo influido por su microentorno se socializa en valores destacadamente «modernos» tenderá a reajustar sus patrones de religiosidad e ideología política (quizás heredados de su familia) en la medida que afectan a su progresiva asimilación de valores modernos. Pero si sus convicciones religiosas son muy fuertes, ejercerán un importante freno y control en la asimilación de los valores modernos. Por lo tanto, en la simulación social basada en agentes, más que definir ecuaciones (también se puede hacer) se trata más bien de definir características y reglas de actuación basadas en dichas características, las cuales pueden ir evolucionando y cambiando en función de la peripecia vital de cada agente.

En resumen, distinguimos dos aportaciones potenciales principales de la simulación en el campo de las ciencias sociales. Una, verificar si un modelo sociológico es coherente y «funciona» adecuadamente. La otra es superar «virtualmente» las grandes limitaciones de experimentación en estas disciplinas, (resulta difícil, cuando no a menudo inmoral o imposible en la práctica, experimentar con sociedades y seres humanos reales) lo que mejoraría las capacidades explicativas e incluso quizás predictivas de estas disciplinas en general y de la sociología en particular. La consecuencia imaginable (plausible) de usar esta nueva herramienta en la investigación social sería sobre todo la mejora y crecimiento de los marcos teóricos, de aquellos sistemas, procesos y fuerzas sociales que se modelan.

Por último, cabe mencionar el potencial integrador de diferentes perspectivas. Un buen modelado informático precisa y admite todos estos inputs; datos, modelos explicativos, interpretaciones y marcos teóricos. Es decir, admite todo tipo de conocimientos de las características del sistema social real. De ahí su capacidad de integrar no solo diversas perspectivas metodológicas (cuanti/cuali) sino también teoría y empiria. Su aplicación adquiere especial sentido cuando en un determinado contexto de investigación existe una cierta abundancia de datos cuantitativos, cualitativos, observaciones longitudinales o transversales, interpretaciones, elaboraciones teóricas constatadas, etc., y queremos (y necesitamos) ir más allá de las limitaciones propias de cada fuente de datos y metodologías, en nuestro intento por mejorar la compresión, explicación y (en lo posible) la predicción de los fenómenos estudiados. Si el conocimiento del sistema a modelar fuese pobre, los resultados también lo serían. No sería aconsejable el modelado informático en estas circunstancias. Por el contrario en situaciones de abundancia de información, estamos en condiciones de elaborar un buen modelado y simulaciones realistas. 


\section{BIBLIOGRAFÍA}

ArRoyo MenÉndez, M. (2004): Cambio Cultural y cambio religioso. Tendencias y formas de religiosidad en la España de fin de siglo. UCM. Madrid.

- (2004b): «Hacia una espiritualidad sin Iglesia». En Tendencias en identidades, valores y creencias. J. F. Tezanos (Ed.). Ed. Sistema. Madrid.

- (2005): «Religiosidad centrífuga: ¿un catolicismo sin Iglesia?». En Iglesia Viva. N. ${ }^{\circ}$ 222, pp. (111-119). Valencia.

AxElrod, R.(1997): «Advancing the Art of Simulation in the Social Sciences». En Conte, R., Hegselman, R., and Terna, P. (eds.). Simulating Social Phenomena, Berlin: Springer, pp. 21-40.

AXELROD , R. (1984): The Evolution of Cooperation. New York: Basic Books, AXELROD, R. (1995), «A model of the emergence of new political actors». In N. Gilbert and R. Conte (eds.), Artificial Societies: the Computer Simulation of Social Life. UCL Press, London.

Axtell, R. (2006): Platforms for Agent-Based Computational Economics. Presentation, VII Trento Summer School.

BIN, Yu; SINGH, M.P.; SyCARA, K. (2004): Developing trust in large-scale peer-to-peer systems, Multi-Agent Security and Survivability, 2004 IEEE First Symposium on Volume, Issue, 30-31 Aug. Page(s): 1-10.

Bratman, M. E. (1987): Intention, Plans, and Practical Reason, Harvard University Press, Cambridge, MA.

Burmeister. B., HADDADI. A., \& MATTILYs. G., (1997): Application of multi-agent sistems in traffic and transportation. Software ingeneering IEE Proceedings. Vol. 114. Issue 1, pp. 51-60.

Castelló, L., Eguíluz, San Miguel (2007): The fate of bilingualism in a model of language competition, Advancing Social Simulation: The First World Congress. Takahashi, Shingo; Sallach, David; Rouchier, Juliette (Eds.), Springer.

CAPCARRERE, M.; SiPPER, M.; TOMASSINI, M. (1996): «Two-state, $r=1$ cellular automaton that classifies density». Phys. Rev. Lett. 77: 4969. doi: 10.1103/Phys. Rev. Lett. 77: 4969.

Cederman, L. E. (2003): Endogenizing Geopolitical Boundaries with Agent-Based Modeling, Proceedings of the National Academy of Sciences, vol. 99, suppl. 3.

DAVIS, M. D. (1971): Introducción a la teoría de juegos. Alianza Editorial. Madrid.

El Hadouaj, S., Drogoul, A. and Espié, S. (2001): «How to Combine Reactivity and Anticipation: The Case of Conflicts Resolution in a Simulated Road Traffic». in: Multi Agent Based simulation. Edited by Scott Moss and Paul Davidsson. Berlin.

GARDNER, M. (1970) «Mathematical Games - The fantastic combinations of John H. Conway's new solitaire game Life,» Scientific American 223, pp. 120-123.

GILBERT, N. and TroITZSCH, K.G. (2006): Simulación para las ciencias sociales. McGraw Hill. Madrid.

GómEZ-SANZ, J. and PAVON, J. (2003): «Agent oriented software engineering with INGENIAS». In: 3rd International Central and Eastern European Conference on MultiAgent Systems, CEEMAS 2003, June 16-18, 2003, Prague, Czech Republic.

JorION, P. (2006): «Adam Smith's Invisible Hand Revisited: An Agent-Based simulation of the New York Stock Exchange,» Proceedings of the First World Congress on Social Simulation Vol. 1: 247-254. Kyoto, August 2006. Berlin: Springer Verlag.

Halman, Ester, De MoOr. (1994): The individualizan society. Value change in Europe

EMPIRIA. Revista de Metodología de Ciencias Sociales. N. ${ }^{\circ}$ 14, julio-dicembre, 2007, pp. 139-161.

ISSN: 1139-5737 
and North America. Tilburg University Press. Tilburg.

Hanneman, R. A. (1988): Computer-Assisted Theory Building. Modelling Dynamic Social Systems. Sage, Newbury Park, CA.

Helbing, D. (1994): Quantitative Sociodynamics. Stochastic Methods and Models of Social Interaction Processes. Kluwer, Dordrecht.

Huhns, M. N. and Singh, M. P. (Eds.) (1997): Readings in Agents. Morgan Kaufmann, San Francisco, CA.

INGLEHART, R. (1977): The silent revolution. Changing values and politica styles among western publics. Princeton University Press. Princeton, New Jersey.

- (1991): El cambio cultural en las sociedades industriales avanzadas. CIS. Siglo XXI. Madrid.

- (1998): Modernización y postmodernización. El cambio cultural, económico y político en 43 sociedades. CIS. Siglo XXI. Madrid.

Kreutzer, W (1986): System Simulation. Programming Styles and Languages. AddisonWesley, Sydney.

LóPez-Paredes, A. Hernández, C., PAjares, J. (2002): «Towards a New Experimental Socio-economics. Complex Behaviour in Bargaining». Journal of Socioeconomics. Vol. 31, pp. 423-429.

LoZARES, C. (2004) «La simulación social. ¿Una nueva manera de investigar en ciencia social?». Papers, revista de sociología n. ${ }^{\circ}$ 72, pp. 165-188.

Pavon, J. Arroyo, M. Hassan, S. y Sansores, C. (2006). «Simulación de sistemas sociales con agentes software», en Actas del Campus Multidisciplinar en Percepcion e Inteligencia, CMPI-2006, volumen I, 389-400.

RAILSBACK, LyTINEN, and JACKSON (2006): Agent-based Simulation Platforms: Review and Development Recommendations. Simulation, September 1, 82(9): 609-623.

Shannon, R. F. (1975): Simulation: A Survey with Research Suggestions, AIIE Transactions. Vol. 7, No. 3, Sept. 1975.

ToBiAs, R. and HofmanN, C. (2004): «Evaluation of free Java-libraries for social-scientific agent based simulation». Journal of Artificial Societies and Social Simulation, vol. 10 .

TroitzSCH, K. G. (1997): «Social Simulation - origins, prospects, purposes». In R. Conte, R. Hegselmann and P. Terna (eds), Simulating Social Phenomena. Lecture Notes in Economics and Mathematical Systems, Vol. 456, pp. 41-54. Springer-Verlag, Berlin.

XIONG and PEERTRUST (2004): Supporting reputation -based trust in peer-to-peer communities. IEEE Transactions on Knowledge and Data Engineering (TKDE), Special Issue on Peer-to-Peer Based Data Management, 2004.

YGGE and AKKERMANS (1995): «Power load management as a computational market». Book title: Proceedings of the first international conference on multi-agent systems. MIT Press. Editor Victor Lesser.

Von Neumann, J. (1966): The Theory of Self-reproducing Automata. A. Burks, ed., Univ. of Illinois Press, Urbana, IL.

ZADEH, L. A. (2006): «Fuzzy sets, fuzzy logic and fuzzy systems». World Scientifics Pub CO Inc. 


\section{RESUMEN}

La simulación social basada en agentes constituye hoy una alternativa para avanzar en el conocimiento sociológico desde una perspectiva metodológica novedosa, poseedora de una potencial vocación integradora. Su aplicación adquiere especial sentido cuando existe una cierta abundancia de datos cuantitativos, cualitativos, observaciones longitudinales o transversales y queremos (y necesitamos) ir más allá de las limitaciones propias de cada fuente de datos y metodologías, en nuestro intento por mejorar la compresión, explicación y (en lo posible) la predicción de los fenómenos estudiados. Además nos ofrece la posibilidad (latente) de articular más y mejor teoría y «empiria», una de las asignaturas pendientes de la sociología, ya que también permite realizar experimentos para verificar hipótesis, examinar supuestos o comprobar la veracidad y coherencia de los marcos teóricos. Los firmantes son miembros del Grupo de Agentes Software, Ingeniería y Aplicaciones (GRASIA) de la UCM y están trabajando en el modelado de un proceso social; las transformaciones religiosas de la sociedad española desde 1980. El artículo ilustrará desde esta experiencia y desde el ejemplo de esta aplicación concreta, las posibilidades, expectativas y también los desafíos, dificultades y limitaciones de la simulación con agentes en la investigación sociológica

\section{PALABRAS CLAVE}

Simulación social. Agentes software. Sistemas multi-agente. Modelos sociales. Sistemas sociales. Metodología

\section{ABSTRACT}

The Agent Based Social Simulation (ABSS) is today a clear alternative for advancing in the sociological knowledge from a new methodological perspective which has a incorporating vocation power. Its application acquires special meaning when there is a big amount of data (quantitative, qualitative, longitudinal or transversal observations) and we want (and need) to arrive further than the constrains of each source and methodology. This will be useful in our attempt to improve the understanding, explanation and (when possible) prediction of the studied phenomena. Furthermore, it offer us the latent possibility of articulating more and better theory and «empiria», one of the failing subjects of Sociology, because it allows to realize experiments for verifying hypothesis, examine assumptions or test the veracity and coherence of the theoretical frames. The signers are members of the Group of Software Agents, Engineering and Applications (GRASIA) of the UCM and they are working in the modelling of a social process: the religious transformations of the Spanish society since 1980. The ar- 
ticle will show from this experience and from the example of this specific application, the possibilities and expectations, together with the challenges, difficulties and limitations of the agent based simulation in the sociological research.

\section{KEY WORDS}

Social simulation. Software agents, Multi-agent systems. Social models. Social systems. Methodology. 